\title{
Several Considerations about Quantum Mechanics
}

\author{
Zhonggang Li \\ Technology Department, Silkworm Research Institute, Anqiu, China \\ Email: 13465701349@163.com
}

How to cite this paper: Li, Z.G. (2021) Several Considerations about Quantum Mechanics. World Journal of Mechanics, 11, 53-70.

https://doi.org/10.4236/wjm.2021.114005

Received: November 17, 2020

Accepted: March 30, 2021

Published: April 2, 2021

Copyright (c) 2021 by author(s) and Scientific Research Publishing Inc. This work is licensed under the Creative Commons Attribution International License (CC BY 4.0).

http://creativecommons.org/licenses/by/4.0/ (c) (i) Open Access

\begin{abstract}
This article has demonstrated the possibility of deriving an equivalent mathematical velocity expression while setting aside the concepts of displacement and time. The proposed velocity formula, better aligned with reality, allows a better understanding of the equivalence of matter and energy, which are comprised of the same type of basic particles. Why, then, is there a significant difference between matter and energy? This is because the combination of the basic particles is not the same. Basic particles are the basic unit of mass and energy, meaning mass and energy conservations are essentially the conservation of these basic particles. Electrons, photons, neutrons, protons, neutrinos, and other microscopic particles also consist of these basic particles. The basic particles are also the basic foundation of force: a basic particle force is the smallest force in the universe, implying that force is not continuous, but a basic particle force is the smallest unit of force. The total mass of a moving body increases with increasing velocities, and this added mass is composed of the basic particles provided by an external system. These basic particles are the foundation of the universe, and determine that physical concepts are vectors or scalars. Velocity, a vector, is the ratio between the basic particles. The concept of time is essentially academic. Although relativity equations may satisfy mathematical principles, they may represent a mathematical model with no physical meaning, not demonstrating objective physical facts.
\end{abstract}

\section{Keywords}

Mass, Energy, Velocity, Concentration, Foron, The Theory of Relativity

\section{Introduction}

With the development of physics, especially the development of production, science and technology since the 19th century, the fact that matter is closely re- 
lated to energy has come to be known. For example, the mass of electrons moving at high speeds increases with the increase of speed, etc.

These facts cannot be explained by classical mechanics as there are contradictions between the theories of classical mechanics and experimental facts. Under these circumstances, the famous physicist Einstein proposed the special theory of relativity in the early 20th century, which marks the beginning of modern physics. The special theory of relativity has fundamentally changed our usual view of space and time and proposed a new concept of time and space. From the perspective of classical mechanics, the length and time interval of an object have nothing to do with the velocity of motion of the object, while the theory of relativity indicates the close relationship between them and speed. The theory of relativity also states that the mass of an object is not fixed and increases with the increase of velocity of motion of the object. Let $m_{0}$ be the mass of an object in the static state, then when the object moves at the velocity of $v$, its mass $m$ can be calculated according to the formula of relativistic mechanics.

$$
m=\frac{m_{0}}{\sqrt{1-\left(\frac{v}{c}\right)^{2}}}
$$

Therefore, classical mechanics is a good approximation of relativistic mechanics at low velocity. As the velocity increases, both mass and kinetic energy increase, and the increase in mass is closely related to the increase of kinetic energy [1].

Einstein obtained the following relationship between mass and energy based on the theory of relativity.

$$
E=m c^{2}
$$

This equation is called mass-energy equation. The equation shows that the mass of an object has a certain relation to its energy: the energy of the object is proportional to its mass. If the mass of an object increases, the energy of the object increases accordingly, and vice versa. In fact, this equation is also consistent with the experiment [2].

In short, we have a full understanding of the close relationship between mass and energy.

"Relativity theory" and "quantum theory" constitute the two fundamentals of modern physics, which represents another development peak of physics after Newtonian mechanics and enables people to quickly carry out research on various aspects of physics. However, modern theories, including relativity theory and quantum theory, are often highly logic and built on rigorous mathematical reasoning represented by successful "mathematical models", but they cannot provide us with intuitive physical prospects.

I believe that time is not a purely physical concept. In physics, the description of velocity with displacement and time is only conclusive and practical but does not indicate its nature. The view of taking time as the basic concept of physics is wrong and it is the cause of leading physics astray. Therefore, the fundamentals 
of physics are unreliable and need to be reviewed from the start of physics several hundred years ago.

Relativity theory was founded on the basis of classical physics, which is an extension of classical physics. Einstein's principle of relativity is idealized, that is to say, Galilean principle of relativity was summarized when an object is moving at low speed, so Einstein reasoned it by analogy in a rash way; Einstein's analogy was to obtain "simultaneity" relativity and "time interval" relativity. In fact, the velocity of light is a consideration that crosses inertial systems. Galilean principle of relativity summarized in low-velocity macroscopic state was arbitrarily analogized to that in high-velocity state as it was, which is unreliable and wrong.

The consideration on the change of mass with velocity and other emerging considerations should be reinterpreted from the view of time and space or nature of matter and energy, which is an either-or thing. This paper focuses on the study of nature of matter and energy rather than space-time view of relativity, holding that matter and energy are essentially the same.

The author believes that matter and energy are unified in nature. In the unity of matter and energy, the concept of "foron" needs to be proposed. (Force is made up of particles. The forces are not continuous. Each particle is one foron). This concept means that matter and energy are just the expressions of foron at different times. A simple foron indicates energy and forons form matter by combining in a certain way. Matter represents "solidified" energy and energy represents "decomposed" matter. Foron is the foundation of matter, energy and force, also the basis of various physical laws. The properties of foron are also the basis of various properties. In short, the world is essentially made up of forons. In this way, a number of questions can be satisfactorily explained. For example, the fact that the mass of an object increases with the increase of its motion velocity can be clearly explained by the theory of foron: the kinetic energy increases with the increase of velocity, that is, the total amount of forons in the object increases; the increase in the forons also means an increase in matter and thus the mass is also increased. Of course, it is only a brief explanation. For another example, mass loss can also be explained from the view of foron: part of matter constituting nucleon is resolved and releases energy, i.e. simple forons. The total amount of forons is reduced, so mass loss occurs after energy release.

The introduction of the concept of "foron" is necessary. As the scope of human knowledge continues to expand, a large number of things that cannot be directly perceived appear in front of us. In this case, we shall assume and design a model which can correctly represent the things being studied within a certain range if the prediction results of the model are consistent with experimental results and its theory completely squares with the facts. In terms of the concept of "foron", if the theory of foron established on the basis of it completely squares with the facts, and all calculation results are completely consistent with experimental results, then we can firmly believe that forons exist within a certain range. Because the concept of matter is quite different from that of energy and 
unaccepted theories in history such as "caloric theory" have misled people, we think that the unity of the two concepts is unbelievable and some people even ridiculed it without judgment. However, we are psychologically resistant to the unity of the two only due to the constraints of definitions and rules made by ourselves as well as our thinking inertia. Then, it is completely achievable for us to rethink the nature of matter, energy and force if we temporarily take a step back hundreds of years to think, which is scientific and necessary, which at least provides us with a new perspective to re-examine the dark matter, dimensionality of the universe, superstring theory, quark, etc. This attempt is beneficial and harmless. In fact, these concepts have been formed after continuous revisions in modern times.

In short, the theory of foron believes that the mass of an object is different when it is in motion and at rest and when the temperature increases and decreases, which has a material basis. The transfer-in and transfer-out of forons will inevitably lead to a change in mass. Certainly, it is not easy to detect such changes under normal circumstances. But sometimes, such as in the state of high velocity, such changes must be considered. That is to say, the conservation of mass and the conservation of energy should be treated in a unified way under certain circumstances, i.e. the conservation of total forons. In fact, we have already done so at certain times, such as the handling of the mass loss problem, which, however, are all limited within a certain range and lack further exploration in theoretical knowledge.

Then, what are the properties of a foron?

Here, let us make simple assumptions and explanations.

1) The action of forces between objects is a property of foron. As the name suggests, force is the property of a foron or a number of forons under certain conditions. The properties of the force of each foron represent the smallest unit of force and a foron is a vector.

2) Foron is energy. A single foron represents the minimum unit of energy. The process of doing work is the process of transporting forons.

3) Forons are combined in a certain way under certain conditions to form matter. Therefore, matter can also be referred to as foron network. After forons are combined into foron networks, they generally do not present the properties of force, but forons (i.e. energy) can be absorbed or released from the foron networks when they are broken up or combined.

4) Foron is also a fundamental factor of motion. A foron is a vector and always goes straight at a speed equal to the velocity of light. A photon is a collective body of forons that are temporarily combined, so the velocity of light is actually the velocity of the forons. It is the purest and most basic velocity, and the maximum velocity in the universe with a value of 1 .

5) Foron indicates the minimum unit of both mass and energy. Foron is the origin of mass and energy.

6) Forons must meet certain conditions to form foron networks. Some forons 
via simple combination also have certain properties of foron.

Foron represents the most basic and smallest unit in the universe, so everything is composed of forons. However, forons can not be directly observed due to their extremely small size.

In short, forons either move forward in a straight line at the velocity of light in a certain way (such as agglomerating into photons), or combined into a foron network to form a new foron network, i.e. matter. In other words, forons tend to combine into foron networks.

It is very monotonous that there are only forons rather than foron networks in the universe. The original universe may be like this, but the universe has become colorful since forons have formed foron networks. At a particular moment (the moment assumed when the universe began), forons first formed some basic foron networks, i.e. many microscopic particles, which were relatively stable and then foron networks such as atoms, molecules, and so on formed due to the further action between the microparticles and forons. Various complex foron networks connect and constitute a unified world of matter. That is to say, those elementary particles are not dots, but constitute the structure of $3 \mathrm{D}$ foron networks.

The properties of a foron network are very different from those of a foron. They become more complex and are displayed in several aspects:

1) A foron network can be disassembled or combined to absorb or release forons, but the total amount of forons in the system is constant. In a certain sense, matter and energy can transform to each other.

2) Forons can be transported via foron network, but when the foron network is not powerful enough to transport a large number of forons and reflect these forons, it will be destroyed to form a new foron network to adapt to the transporting and reflection of a large number of forons, or absorb or release forons in the process of forming a new foron network.

3) Mass represents the total amount of forons that constitute foron networks. Under the same force conditions, if the mass is the same, the amount of forons absorbed from external force is also the same.

4) Forons that act on an object with a certain regularity in a certain order, we call them ordered forons; if many forons act on an object irregularly, we call them disordered forons. Although both ordered forons and disordered forons form new foron networks after entering an object, their macroscopic performance is different. For example, motion is caused by ordered forons and heat energy is caused by disordered forons.

5) The so-called anti particle and anti matter are composed of forons via another unstable combination.

Therefore, the nature of the law of conservation of energy and the law of conservation of mass is that the total amount of forons is constant, which is very simple and straightforward. At the same time, we realize that the nature of mass is the total forons contained in foron networks. In this way, we can better un- 
derstand that mass is a property of matter itself. However, we can know from the theory of foron described later that, mass changes with the change of velocity and temperature, because the change of velocity and temperature is determined by the increase and decrease of forons. In general, we cannot detect this change as the amount of such increased and decreased forons is too small compared to the amount of forons originally contained in the object.

Matter and energy are unified. Foron is the minimum unit of energy and the only component of matter. The amount of forons contained in the foron networks indicates the mass. Then, the measurement units of energy and mass should be uniform and both are used to indicate the amount of forons. Therefore, the mass unit currently used by us can also be taken as the measurement unit of energy.

In short, after the units are unified, energy and mass can be added and subtracted to each other. For example, an object at rest has a mass of $m$ and kinetic energy of $Q$, then its exact mass when it moves is:

$$
M=m+Q .
$$

The explanations of some physical problems by the theory of foron are more intuitive. For example, when a Y photon with an energy of $2.22 \mathrm{MeV}$ is used to illuminate a deuteron (which consists of a proton and a neutron), it can be broken down into a proton and a neutron. The nuclear reaction equation is as follows:

$$
Y+{ }_{1}^{2} H \rightarrow{ }_{1}^{1} H+{ }_{0}^{1} n
$$

The opposite process also occurs. A proton and a neutron are combined to form a deuteron by releasing an energy of $2.22 \mathrm{MeV}$. This energy is radiated in the form of a Y photon. The nuclear reaction equation is as follows:

$$
{ }_{0}^{1} n+{ }_{1}^{1} H \rightarrow{ }_{1}^{2} H+Y
$$

In the view of the theory of foron, the difference between the two nuclear reactions only indicates the difference between the combination types of forons before and after the reactions, but the total amount of forons before and after the reactions is always equal, that is,

$$
Q_{0}^{1} n+Q_{1}^{1} H \rightarrow Q_{1}^{2} H+Q Y
$$

According to the theory of foron, the annihilation of a pair of positive and negative particles which meet and disappear at the same time and are transformed into other kinds of particles also shows that the combination types of forons before and after the reactions are different, but the total amount of forons is always equal from start to finish. For example, a positron and a negatron will annihilate each other and convert into a pair of photons:

$$
{ }_{1}^{0} e+{ }_{-1}^{0} e=Y+Y
$$

The theory of foron believes that the forons that constitute positive and negative electrons immediately form a pair of photons after the pair of electrons are 
broken down. This example shows to a certain extent that energy and mass are unified and they are forons in different forms. For another example, the forons with an energy of $1.02 \mathrm{MeV}$ can constitute a photon of $1.02 \mathrm{MeV}$ or a positive electron and a negative electron, that is,

$$
\begin{gathered}
Y \rightarrow{ }_{-1}^{0} e+{ }_{1}^{0} e \\
1 U=931.5 \mathrm{MeV}=1.660566 \times 10^{-27} \mathrm{~kg}
\end{gathered}
$$

The amount of forons on the right $=0.91 \times 10^{-30} \mathrm{~kg} \times 2=1.82 \times 10^{-30} \mathrm{~kg}$.

The amount of forons on the left $=1.02 \mathrm{MeV}=1.82 \times 10^{-30} \mathrm{~kg}$, i.e., the amount of forons on the left is equal to that on the right,

$$
Q_{Y}=Q_{-1}^{0} e+Q_{1}^{0} e
$$

The energy on both sides of the equal sign is $1.02 \mathrm{MeV}$, or $1.82 \times 10^{-30} \mathrm{~kg}$.

That is to say, although there are differences between electron and photon, both of them are composed of forons in different forms. Furthermore, forons are equivalent to basic building materials and constitute various microscopic particles.

A photon is a body where forons temporarily aggregate, which are either transformed into a foron network (such as electrons) that exists at rest, or move forward at the velocity of light. The larger amount of forons contained in a photon, the larger the mass of the photon, that is, the energy is also large. On the contrary, it is small, but the velocity is certain. Because photons cannot exist in a static state under normal conditions, we used to say that photons have energy and momentum, which is not comprehensive. Energy and mass are unified, and the amount of forons moving at the velocity of light also indicates the mass. The viewpoint of the theory of foron makes this physical prospect clearer.

Of course, the above only represents a rough view of the theory of foron which is a bold assumption and not perfect at current stage. In addition, other physics theories (such as quantum field theory) have self-contained arguments on the above examples. In fact, there is no conflict between the theory of foron and the quantum mechanical theory in these aspects, because the two theories focus on different problems. The quantum mechanics studies the law of microscopic particle motion in the later stage of microscopic particle formation, while the theory of foron studies problems from the perspective of foron, which is a blank and more microscopic field.

\section{Derivation and Calculations}

\subsection{Consideration of Velocity}

After the world of matter formed, various motions have been produced in the universe. Mechanical motion is the most common phenomenon in the universe. A basic problem in mechanical motion needs to be clarified first, i.e. "What is the cause of motion?"

More than two thousand years ago, the ancient Greek philosopher Aristotle 
believed that force is the cause of maintaining the motion of an object. This statement had been used till the 17th century before the mistakes of it were pointed out by the famous Italian physicist Galileo.

Today, if anyone holds Aristotle's point of view, we will retort upon him in unison, "No! Inertia is the reason for maintaining the motion of an object." This statement is correct. However, if we make two identical objects move, one moves at a uniform velocity in a straight line and the other stands still, then what are the differences between the two objects? That is, since both of them are maintained by inertia, why are they in different states? What is the nature of inertia?

According to the theory of foron, velocity is a property of a moving object itself and has nothing to do with displacement and time. Time is not a purely physical concept. The concept of time can be used in production and life, but cannot be used to describe velocity when studying the basic problems of physics. Both classical physics and relativity theory describe velocity with displacement and time. Therefore, the disagreements between the theory of foron and them are essential differences at the starting point of physics hundreds of years ago.

Ordered forons enter the object sequentially and form new foron networks with original foron networks of the object. The properties of the new foron networks are no longer the same as those of the stationary object. Therefore, the object moves due to the incoming of the forons.

We know from the properties of a foron that a foron moves in a linear direction and the object is in linear motion from static state, which can only be determined by the properties of a foron. In the absence of external force, the velocity of a moving object is related to the amount of ordered forons entering the object. If the amount of forons does not change, the foron networks of the moving object remain unchanged, that is, the velocity and direction remain unchanged.

In short, both motion and rest are properties determined by foron network structure. The magnitude of the velocity is related to the amount of forons entering the object.

Since velocity is a property of foron networks of an object, the magnitude of the velocity is determined by the amount of ordered forons and the direction of the velocity is also determined by the direction of the forons. Then, how to calculate and measure the magnitude of the velocity?

Because the magnitude of the velocity is determined by the amount of ordered forons and the amount of forons represents mass, the velocity should be calculated and measured by mass.

How do we express the velocity? We know that a moving object $M$ contains the rest mass $m$ and the incoming forons $Q$, i.e. $M=m+Q$, therefore, we can only use these measures to express the velocity. $Q$ The unit of is kilogram, which is consistent with the units of $M$ and $m$. Meanwhile, we specify the velocity of a foron as the basic unit of velocity with a value of 1 .

The theory of foron believes that, the velocity $V$ of a moving object is the ra- 
tio of kinetic energy $Q$ to the total forons $(m+Q)$ of the object, similar to the concept of "concentration", which is a property of foron networks of the object after obtaining such energy and has nothing to do with others, that is, the "concentration" of $Q$ is large and the velocity also increases; on the contrary, the velocity decreases. That is, $Q$ is equivalent to "solute", $m$ is equivalent to "solvent", and "velocity" is a property of the "solution" $M$ formulated by them. This is the understanding of nature of velocity according to the foron theory.

The proposed formula is as follows:

$$
V=\frac{Q}{m+Q} \cdot X
$$

where $X$ is a coefficient.

This is similar to the formula calculating the concentration of a solution. The percentage concentration of a soluble substance in a solvent forming a solution (the mass fraction of the solution), known as the solution percentage concentration, is expressed as:

$$
\text { Concentration } \cdot \mathrm{C}=\frac{m(\text { solute })}{M(\text { solvent })+m(\text { solute })} \times 100 \%
$$

$X$ is a factor. Considering that this ratio cannot be simple, a coefficient of $X$ is required. It can be seen from this velocity formula that when $m_{0}$ is certain, there are more forons entering the object and the velocity increases; on the contrary, the velocity decreases. Therefore, the velocity formula must at least meet the following conditions with reference to the concept of concentration:

1) When, $m \neq 0, Q=0, V=0$.

2) When $m \neq 0, Q>0, V=1$, the velocity is at a maximum value of 1 .

3) When, $m \neq 0, Q>0, V$ increases with $Q$, but never reaches 1 ; $V$ decreases with $Q$ but is always greater than zero. That is, $0<V<1$.

The theory of foron believes that, there were only forons in the original universe at a certain stage. The motion at the velocity of light is a property of forons. Foron is the foundation of force, mass, energy and velocity. In the new world formed later, velocity does not indicate purely velocity of light, but fast or slow velocity. The combination of ordered forons and foron networks into new foron networks is slower than the velocity of a single foron. The maximum velocity in the universe is the velocity of a simple foron and numerically equal to the velocity of light. We specify it as the basic unit of velocity with the value of 1 .

Therefore, (with reference to the derivation process Of Li, Zhonggang: Reflections on the Direction of Physics. American Academic Press, America (2020) Chapter 2), the velocity formula of the foron theory is as follows:

$$
\begin{aligned}
V & =\frac{Q}{m+Q} \cdot X=\sqrt{\frac{Q^{2} X^{2}}{(m+Q)^{2}}} \\
& =\sqrt{\frac{Q^{2} X^{2}}{m^{2}+2 m Q+Q^{2}}}
\end{aligned}
$$

From the derivation, we can see that the formula can meet all three of the 
above requirements only when $Q^{2} X^{2}=2 m Q+Q^{2}$. Therefore,

$$
\begin{gathered}
Q^{2} X^{2}=2 m Q+Q^{2} \\
X^{2}=\frac{2 m Q+Q^{2}}{Q^{2}} \\
X^{2}=\frac{2 m}{Q}+1 \\
X=\sqrt{\frac{2 m}{Q}+1}
\end{gathered}
$$

Therefore, the velocity formula can be expressed as follows:

$$
V=\frac{Q}{m+Q} \cdot \sqrt{\frac{2 m}{Q}+1}
$$

Other equivalent forms of the above equation include

$$
\begin{gathered}
V=\sqrt{\frac{2 m Q+Q^{2}}{m^{2}+2 m Q+Q^{2}}} \\
V=\sqrt{\frac{2 Q+\frac{Q^{2}}{m}}{m+2 Q+\frac{Q^{2}}{m}}} \\
V=\sqrt{\left.\frac{Q(M+m)}{M^{2}} \text { (where } M=m+Q\right)}
\end{gathered}
$$

Therefore, it can be seen that the velocity $V$ is a certain ratio of $Q$ to $\left(m_{0}+Q\right)$, which indicates a mass relation, similar to the concept of "concentration" [3].

If we assume that the maximum possible speed of 1 is the speed of light, all applications and calculations show that the results from this extremely simple starting point - the velocity equation-are completely consistent with reality. Remarkably, the results are consistent with relativity-based calculations! Whereas relativity-based formulas are derived from complicated space-time relationships, our formula was derived simply and intuitively by considering the concentration of a solution. Although the starting points are different, the calculated results are the same. The following simple comparison calculations verify this fact:

1) Assume an object with high velocity. When $M=1.7 \mathrm{~m}$ and the object's resting mass $m=1$, so that the additional mass of 0.7 comes from $Q$, what is the velocity? According to relativity-based calculations, $V \approx 0.8 c$, that is, $80 \%$ of the speed of light.

Using the velocity equation presented above, the result is:

$$
\begin{aligned}
V & =\frac{Q}{m+Q} \cdot \sqrt{\frac{2 m}{Q}+1}=\frac{7}{17} \times \sqrt{\frac{27}{7}} \\
& \approx 0.8(\text { or } 0.8 c)
\end{aligned}
$$

Hence, the results are the same.

Assuming that the maximum value of 1 is equal to the speed of light, if $V \approx 0.8$ 
and we use $3 \times 10^{8} \mathrm{~m} \cdot \mathrm{s}^{-1}$ as the speed of light, then $V \approx 2.4 \times 10^{8} \mathrm{~m} \cdot \mathrm{s}^{-1}$. The magnitude of $V$ depends on the specific units we use, which are arbitrary. The units only exist as tools that people use for convenience.

2) Assume an object moving at high velocity. When $M=1.25 \mathrm{~m}$, what is the velocity? Using relativity-based formulas, we can arrive at $V=0.6 c$.

Using the velocity equation presented above, we get

$$
\begin{aligned}
V & =\frac{Q}{m+Q} \cdot \sqrt{\frac{2 m}{Q}+1} \\
& =\frac{1}{5} \times \sqrt{9} \\
& =0.6(\text { or } 0.6 c)
\end{aligned}
$$

We can see that the results of the two calculations are the same.

We can see that matter and energy are equivalent; then the velocity formula presented in this paper only expresses the proportional relationship between basic quantum quantities. Only by setting the velocity of 1 equal to some specific value, the formula gives us values corresponding to real-world units. For convenience, we can make substitutions in common concepts from modern physics. In this theory, matter and energy are equivalent, and are composed of the same type of basic particles. Therefore, I have set a temporary rule; the standard units of mass and energy are the same, i.e., kilogram, and the maximum speed is 1. In essence, speed is a ratio, and it does not have units, but a maximum value of 1 , which is the speed of light. Hence, the calculation can give us values corresponding to real world units. The unit conversion is in full compliance with existing mathematical and physical laws.

Now, let us derive a formula for energy (we already know that $M=m+Q$ ).

According to the velocity formula

$$
V=\frac{Q}{m+Q} \cdot \sqrt{\frac{2 m}{Q}+1}
$$

Therefore,

$$
\begin{aligned}
& V^{2}=\frac{Q^{2}}{M^{2}} \cdot \frac{2 m+Q}{Q} \\
& M^{2} V^{2}=Q(2 m+Q) \\
& M^{2} V^{2}=Q(M+m)
\end{aligned}
$$

Thus, the energy formula is

$$
Q=\frac{M V^{2}}{1+\frac{m}{M}}
$$

We can draw the following three conclusions:

1) Under low-speed conditions, $M \approx m$. That is, $1+\frac{m}{M} \approx 2$. Therefore, 


$$
\begin{array}{r}
Q=\frac{M V^{2}}{1+\frac{m}{M}} \\
Q \approx \frac{1}{2} M V^{2}
\end{array}
$$

Here, we were able to derive the classical physics formula for kinetic energy without relying on the concepts of displacement or time. We can also see that this formula only applies to low-velocity conditions. The formula for kinetic energy in classical physics is based on a mechanical calculation. However, classical physics was developed to describe a low-velocity world, where this formula is accurate enough and its practical importance cannot be discounted.

2) Under high-speed conditions, we must consider the effects of changing mass. Thus, we get a dynamic energy equation, not a mechanical one.

$$
Q=\frac{M V^{2}}{1+\frac{m}{M}}
$$

3) When $m=0$, and $M \neq 0,1+\frac{m}{M}=1$. Therefore,

$$
Q=\frac{M V^{2}}{1+\frac{m}{M}}=M V^{2} \text { and } Q=M c^{2}
$$

We can see that the derived energy equation is equivalent to the mass-energy equation of relativity.

During the application of the energy equation, if we assume that the highest possible speed of 1 is equal to the speed of light (with its units), then the unit for both mass and energy is the kilogram. However, a kilogram represents an enormous amount of energy. The earth receives approximately $2 \mathrm{~kg}$ of energy from solar radiation every second, which is enough to drive many natural phenomena and sustain all life on earth. Therefore, the kilogram is not an appropriate unit for discussing the quantities of energy we encounter in daily life. If we use $\mathrm{m} \cdot \mathrm{s}^{-1}$ as the unit for velocity, then the unit for energy is Joule, which is more convenient to use. In essence, the specific units we use for energy depend on the number of parts into which we divide the maximum velocity.

\section{Verification of formula}

Using the velocity formula derived above, we can also derive another energy formula. Using another form of the velocity equation from above:

$$
\begin{gathered}
V=\sqrt{\frac{2 m Q+Q^{2}}{(m+Q)^{2}}} \\
V^{2}=\frac{2 m Q+Q^{2}}{(m+Q)^{2}} \\
m^{2} V^{2}+2 m Q V^{2}+Q^{2} V^{2}=2 m Q+Q^{2}
\end{gathered}
$$




$$
\begin{aligned}
Q & =\frac{\left(V^{2}-1\right) Q^{2}+\left(2 m V^{2}-2 m\right) Q+m^{2} V^{2}=0}{2 a} \\
= & \frac{-\left(2 m V^{2}-2 m\right) \pm \sqrt{\left(2 m V^{2}-2 m\right)^{2}-4\left(V^{2}-1\right) m^{2} V^{2}}}{2\left(V^{2}-1\right)} \\
= & -m \pm \frac{2 m \sqrt{1-V^{2}}}{2\left(V^{2}-1\right)} \\
= & -m \pm \frac{m \sqrt{1-V^{2}}}{V^{2}-1}
\end{aligned}
$$

Since $Q$ cannot be negative, we eliminate one of the roots to get

$$
\begin{aligned}
Q & =-m-\frac{m \sqrt{1-V^{2}}}{V^{2}-1} \\
& =-m+\frac{m \sqrt{1-V^{2}}}{\sqrt{1-V^{2}} \sqrt{1-V^{2}}}
\end{aligned}
$$

and

$$
Q=\frac{m}{\sqrt{1-v^{2}}}-m
$$

The total mass $M=m+Q$; hence,

$$
M=\frac{m}{\sqrt{1-v^{2}}}
$$

In the theory of relativity, we compare the relationship between moving mass and rest mass during the object's motion

$$
M=\frac{m}{\sqrt{1-\left(\frac{v}{c}\right)^{2}}}
$$

In the formula in this paper, $v$ is the speed of light with a maximum value of 1. The numerical values are compared, and the relativistic mass formula $\left(\frac{v}{c}\right)$ is found to be the same. Therefore, the relativistic mass formula for $v^{2}-\left(\frac{v}{c}\right)^{2}-$ is the same. Hence, the formula in this paper is the same as the formula of relativity. Because the relativity formula has been verified for real situations, the formula presented in this paper is also validated.

This formula is convenient for use in certain calculations. For example, what is the energy of a body of mass $m=1 \mathrm{~kg}$ with a velocity $V$ of $0.6 \mathrm{c}$ ? If we substitute $V=0.6$ c into the formula, we get

$$
Q=\frac{m}{\sqrt{1-V^{2}}}-m=0.25 \mathrm{~kg} \text { and } M=1.25 \mathrm{~kg} \text {. }
$$

This formula is extremely similar to the relativity-based formula and the ob- 
tained result is also the same.

The theoretical basis of the theory of foron is different from that of the theory of relativity. The velocity formula is directly derived for the first time combined with physical facts according to the foron theory based on the concept of "foron" and with reference to the concept of "concentration". The theory of relativity is based on the relativistic view of time and space. In the formula derivation, the complex time relation is first studied, and then the relevant formula is derived. With respect to the same results calculated with the formulas, I don't think it is a coincidence. They indicate different descriptions on the same problem in the same physical world by the foron theory and the relativity theory from different angles. The related calculations of relativity are based on a fictitious route of light propagation. In short, the theory of foron is an intuitive physical view based on the concept of "foron", and the theory of relativity is a mathematical physical work established by deduction. I believe that mathematics is a rigorous science and physics cannot be separated from mathematics. However, we cannot think about the physical world relying entirely on mathematics. Otherwise, our thinking on the real physical world may completely slide into the established way of thinking, bound by numbers, and even become a digital game.

The proposed approach is able to solve relativity-related problems, and can obtain a simple, intuitive, and unified picture of physics with some further development. The basic particle, as the basic unit of mass and energy, represents the smallest possible increment of mass and energy. Mass and energy conservation thus essentially represent the conservation of these basic particles. Electrons, photons, neutrons, protons, neutrinos, and other microscopic particles are composed of these basic particles. The basic particle is also the basic foundation of force, representing the smallest increment of force in the universe. This implies force is not continuous, but consists of basic particles of force. The function of force is simply a property of basic particles. The proposed system transfers basic particles by force or radiation.

\subsection{About Quantum Mechanics}

We should first discuss the considerations about photon.

There are many complicated considerations involving photon, including electromagnetic field and other issues which we will discuss later. Now let us discuss photons separately.

A photon is made up of forons. Since a photon is the aggregation of forons, it will fully reflect the properties of foron. For example, it will move forward at the velocity of forons, i.e. the velocity of light. Of course, a photon is still different from a foron. Under certain conditions, forons contained in a photon can be transferred to other forms of energy such as heat, and then the photon does not exist. Under specific conditions, forons contained in a photon may also form new particles and then the photon disappears.

Facts have proved that photons exhibit wave-particle duality and their fluctua- 
tions are perplexing. In 1900, German physicist Planck discovered when he studied the law of electromagnetic radiation that calculation results are fairly consistent with experimental facts only by assuming the energy of electromagnetic radiation is discontinuous, but piece by piece, and every piece of energy is $h v$, where $v$ is the radiation frequency and $h$ is a universal constant. Inspired by Planck's quantum theory, Einstein boldly predicted when he studied the photoelectric effect that light is transmitted piece by piece and every piece of light is called a photon. The energy of a photon is proportional to its frequency, i.e. $E=h v$, where $h$ is the Planck constant. Einstein made this judgment in the case of insufficient experimental facts, but all the results from this assumption are consistent with the results of subsequent experiments.

From Formula $E=h v$, we can see that the higher the frequency of a photon, the larger its energy. The energy of a photon can also be calculated based on the formula: $E=m c^{2}$. Since both are the formulas of energy of a photon, thus,

$$
m c^{2}=h v
$$

The following formula can be derived by further conversion.

$$
\lambda=\frac{h}{p}
$$

It is the formula of light wavelength. In fact, this formula has already been derived.

Inspired by the wave-particle duality of light, French physicist De Broglie proposed a hypothesis in 1924 pointing out that not only photons but also all microscopic particles, including electrons, protons and neutrons have the wave-particle duality. He extended the photon wavelength formula $\lambda=\frac{h}{p}$ to all microscopic particles, pointing out that the moving particles with mass of $\mathrm{m}$ and velocity of $v$ also have wave properties with the same wavelength formula of $\lambda=\frac{h}{p}$. This formula is fairly consistent with experimental facts [4].

From Formula $E=h v$, we can see that if the frequency of a photon is high, its energy will be large. When we analyze the cause of photon wave behavior today, we can conversely assume that the energy of a photon is large so its frequency is high. Forons contained in a photon is the fundamental cause of photon wave and the amount of forons determines the frequency of photon wave. This is the viewpoint of the theory of foron.

The theory of foron believes that, the microscopic world and the macroscopic world are unified. Like the nature of velocity studied above, the fluctuations of micro-particles such as electrons, protons and neutrons are also determined by the amount of forons entering them. We will discuss the formulas of fluctuations of these particles: energy formula, wavelength formula and frequency formula.

A particular particle moves at a certain velocity and its motion is unique. That is, its energy, frequency, wavelength, etc. are unique. Therefore, a factor of $H$ can always be found, and the product of $H$ and frequency $V$ is equal to the energy $Q$, i.e. 


$$
Q=H V
$$

As the frequency is $V=\frac{v}{\lambda}, \lambda=\frac{h}{m v}$, so, $V=\frac{m v^{2}}{h}$, then the following formula can be obtained.

$$
\begin{gathered}
Q=H V \\
Q=H \frac{m v^{2}}{h}
\end{gathered}
$$

According to the formula of kinetic energy in the foron theory,

$$
Q=\frac{m v^{2}}{1+\frac{m_{0}}{m}}
$$

Thus,

$$
\frac{m v^{2}}{1+\frac{m_{0}}{m}}=H \frac{m v^{2}}{h}
$$

By arranging, we can obtain the formula as follows.

$$
H=\frac{h}{1+\frac{m_{0}}{m}}
$$

where, we can see that the kinetic energy $Q$ of a particle (such as a moving electron) is equal to the product of $\frac{h}{1+\frac{m_{0}}{m}}$ and frequency $V$. That is,

$$
Q=\frac{h}{1+\frac{m_{0}}{m}} \cdot V
$$

it is a formula through exact calculation. Generally speaking, it can be divided into three conditions:

1) In a low velocity motion, $m \approx m_{0}, 1+\frac{m_{0}}{m} \approx 2$, then

$$
\begin{gathered}
H=\frac{h}{1+\frac{m_{0}}{m}} \approx \frac{h}{2} \\
Q \approx \frac{1}{2} h V
\end{gathered}
$$

For example, when an electronic moves at the velocity of $8.4 \times 10^{6} \mathrm{~m} / \mathrm{s}$, its kinetic energy is

$$
Q \approx \frac{1}{2} h V=3.2 \times 10^{-17} \text { joules }
$$

2) When the velocity is close to the velocity of light, then

$$
\frac{1}{2} h<\frac{h}{1+\frac{m_{0}}{m}}<h
$$


So, $\frac{1}{2} h V<Q<h V$

3) When the velocity of a photon is $c$, the rest mass is $m_{0}=0,1+\frac{m_{0}}{m}=1$, then

$$
\begin{gathered}
H=h \\
Q=h V
\end{gathered}
$$

That is, $E=h v$

The quantum theory proposed by Planck in 1900 reveals the situation when the velocity of a photon is the velocity of light, the energy of the photon is $E=h v$, which is an exception of the foron theory with regard to the quantum problem.

Through discussion in this paper, we can see that many motion problems are related to the amount of new forons. Those complicated calculations, illustrations and thinking processes need not be listed here. The physics research from macro and micro perspectives has finally achieved perfection and unity.

\section{Conclusion}

This article has demonstrated the possibility of deriving an equivalent mathematical velocity expression while setting aside the concepts of displacement and time. The proposed velocity formula, better aligned with reality, allows a better understanding of the equivalence of matter and energy, which are comprised of the same type of basic particles. Why, then, is there a significant difference between matter and energy? This is because the combination of the basic particles is not the same. Basic particles are the basic unit of mass and energy, meaning mass and energy conservations are essentially the conservation of these basic particles. Electrons, photons, neutrons, protons, neutrinos, and other microscopic particles also consist of these basic particles. The basic particles are also the basic foundation of force: a basic particle force is the smallest force in the universe, implying that force is not continuous, but a basic particle force is the smallest unit of force. The total mass of a moving body increases with increasing velocities, and this added mass is composed of the basic particles provided by an external system. These basic particles are the foundation of the universe, and determine that physical concepts are vectors or scalars. Velocity, a vector, is the ratio between the basic particles. The concept of time is essentially academic. Although relativity equations may satisfy mathematical principles, they may represent a mathematical model with no physical meaning, not demonstrating objective physical facts. The nature of velocity has nothing to do with time or displacement, meaning velocity and space-time are unrelated. A formula was derived simply from this perspective, not relying on the complexities of relativistic spacetime. According to Occam's razor, the simplest explanation may just be the most accurate. 


\section{Conflicts of Interest}

The author declares no conflicts of interest regarding the publication of this paper.

\section{References}

[1] Miller, I. (1981) Albert Einsteins Special Theory of Relativity. Addison-Wesley, London.

[2] Dirac, P.A.M. (2011) General Theory of Relativity. World Book Publishing Company, Beijing.

[3] Li, Z.G. (2020) Reflections on the Direction of Physics. American Academic Press, Salt Lake.

[4] Griffiths, D.J. (2008) Introduction to Quantum Mechanics. China Machine Press, Beijing. 